\title{
Role of amniotic fluid index on maternal and neonatal outcomes among obstetric women with preterm premature rupture of membranes
}

\author{
Jennifer Britto J.*, Sailatha R., Amrita Priscilla Nalini
}

Department of Obstetrics and Gynecology, Chettinad Hospital and Research Institute, Chennai, Tamil Nadu, India

Received: 09 August 2018

Accepted: 04 September 2018

\section{*Correspondence:}

Dr. Jennifer Britto J.,

E-mail: drjenni12345@gmail.com

Copyright: (C) the author(s), publisher and licensee Medip Academy. This is an open-access article distributed under the terms of the Creative Commons Attribution Non-Commercial License, which permits unrestricted non-commercial use, distribution, and reproduction in any medium, provided the original work is properly cited.

\begin{abstract}
Background: Preterm Premature rupture of Membrane (PPROM) is one of the major complications of a pregnant women with risk factors like low socioeconomic class, infections, STDs etc. Maternal complications such as Chorioamnionitis, Abruptio placenta, sepsis and neonatal complications like neonatal sepsis, RDS, intraventricular hemorrhage are common with those with PPROM. The objectives of the present study were to find the association between AFI values and maternal and fetal outcomes in patients with preterm premature rupture of membranes Methods: A cross sectional study was conducted in a Multispecialty Teaching Hospital, North Chennai, Tamil Nadu with the sample size of 100 . The study participants included pregnant women with gestational age of 28 to 34 weeks presenting with PPROM. Maternal outcomes included clinical diagnosis of chorioamnionitis, placental abruption, meconium in liquor, fetal distress, prolapsed cord and mode of delivery. Neonatal outcomes include neonatal hospitalization in NICU, APGAR score at 1st minute and 5 minutes, early neonatal sepsis neonatal death. Significance of difference in means was calculated using independent $t$ test.

Results: Total study participants were divided into 2 groups with Amniotic Fluid Index (AFI) of $>5$ and $<5$. Latency period, Chorioamnionitis, placental abruption and mode of delivery were compared between the groups. Chorioamnionitis was present in $8 \%$ and $12 \%$ in group 1 and group 2 respectively. Neonatal outcomes like NICU admission, RDS and neonatal sepsis were also compared between the groups. NICU admission was required for $18 \%$ and $48 \%$ in group 1 and 2 respectively. APGAR scores at 1 and 5 minutes were found to be lower among those with Group 2. ( $\mathrm{p}=0.000)$.

Conclusions: PROM is associated with increased maternal and neonatal complications. AFI has been proven a role in predicting maternal and neonatal outcomes in PROM. Identifying the risk factor and its treatment remains the main mode of prevention for PROM.
\end{abstract}

Keywords: Abruptio placenta, Chorioamnionitis, NICU, PPROM

\section{INTRODUCTION}

Preterm premature rupture of the membranes (PPROM) is one of the fatal complications during pregnancy with significant perinatal complications. PPROM is defined as the rupture of the amniotic membranes before 37 weeks of gestation and before the onset of labour. ${ }^{1}$ PPROM constitutes one third of the preterm births and it complicates about $3 \%$ of pregnancies. ${ }^{2}$ Predisposing factors of PPROM are low social economic class, previous history of preterm labour, smoking, infections, sexually transmitted diseases, genetic and/or enzymatic abnormalities, nutritional deficiencies, intervention such as circlage and amniocentesis. ${ }^{3-5}$

Major maternal risks are chorioamnionitis (35\%), abruption $(19 \%)$ and sepsis $(<1 \%)$. Abruptio placenta is frequently seen if the rupture of membrane occurred 
before 28 weeks of gestation. ${ }^{6}$ In the presence of intrauterine infection or oligohydromnios, the risk of abruption is high. ${ }^{7}$

Fetal morbidity due to PPROM includes pulmonary hypoplasia, Respiratory Distress Syndrome, sepsis, intraventricular hemorrhage and neonatal sepsis. ${ }^{8}$ Presence of adequate amniotic fluid prevent infections and thereby reducing the occurrence of PPROM. Thus oligohydroamnios is also considered as risk factor for PPROM. ${ }^{9}$ When the presentation is non cephalic in PPROM there are risks such as shorter latency and increased neonatal morbidity, to be increased when oligohydraminos is present. $^{10}$ In addition, there are reports about unfavourable impact by noncephalic presentation of the fetus with PPROM on the antepartum, intrapartum and neonatal risks, which were primarily due to cord prolapse. ${ }^{11}$ The objective of the present study was to find the association between AFI values and maternal and fetal outcomes in patients with preterm premature rupture of membranes.

\section{METHODS}

The study was conducted in a Multispecialty Teaching Hospital located in North Chennai of Tamil Nadu, India. The study was a cross sectional type of study. The study included patients presenting to Labour room with Preterm PROM from 28 to 34 weeks period of gestation (POG). Inclusion criteria was pregnant women with gestational age 28 to 34 weeks presenting with leaking per vaginum. Exclusion criteria includes - symptoms of chorioamnionitis at the time of admission, history of previous caesarean section or previous uterine surgery, non-cephalic presentation, Intrauterine growth restriction, maternal diseases complicating pregnancy such as preeclampsia, diabetes, ecclampsia etc.

The study was carried out between June 2015- October 2016. Sample size was calculated with the formula for cross sectional studies, i.e. $\mathrm{N}=\mathrm{Z} 2 \mathrm{pq} / \mathrm{L} 2$.

The incidence of preterm premature rupture of membranes from previous studies in India ranges from $3.0-10.0 \%$ of all deliveries. ${ }^{12}$ With the incidence of PPROM at $7 \%, Z=1.96$ and error of $5 \%$, the sample size calculated was 100 .

Patients fulfilling inclusion criteria and willing to participate in the study were included successivelyconsecutive sampling method was followed. AFI value of the previous visit was taken to divide the study population into two groups. In the present study, two groups were made-those with AFI $>5$ and those with AFI $<5$. Thus, enrollment was continued till each group consisted 50 patients.

Participants who fulfilled the inclusion criteria were hospitalized, admitted in labour room and are monitored for 12 hours in view of emerging contractions, bleeding or possible start of delivery after NST and fetal heart rate monitoring. Those with the complaints of leaking per vaginum with confirmed leaking were determined by using sterile speculum examination using the pooled fluid and the secretions were examined by fern test under microscopic and litmus paper test. Ultrasonogram was performed on all the participants during first 12 to 24 hours to measure amniotic fluid in four abdominal quadrants in order to determine AFI. Participants were divided into 2 groups according to their AFI. Supportive management such as administration of single course of betamethasone were done to the patients at admission ( 2 doses of inj betamethasone 12mg, 24 hours apart) for fetal lung maturity.

Antibiotic prophylaxis for the participants includes inj ampiliciln 1gram i.v. 6hourly for first 48 hours was given and then followed by cap amoxicilin 500mg 8hourly orally till the delivery. During hospitalization fetal heart rate was monitored for every 2 hours, daily non-stress tests was performed for fetus with gestational age $>28$ weeks.

The patients were observed for clinical symptoms of chorioamnionitis such as fever, uterine tenderness, maternal tachycardia, fetal tachycardia, laboratory symptoms (leucocytosis). If symptoms suggested start of clinical chorioamnionitis, antibiotics were given and if delivery is not started, labour was induced.

Maternal outcomes such as period of latency, clinical diagnosis of chorioamnionitis, placental abruption, meconium in liquor, fetal distress, prolapsed cord and mode of delivery. Neonatal outcomes such as neonatal hospitalization in NICU, duration in NICU stay, APGAR score at 1 st minute and 5 minutes, RDS, early neonatal sepsis and early neonatal death.

Data analysis was done with Statistical Package for Social Sciences (SPSS IBM) version 21.0. The qualitative variables are described in the form of proportions and quantitative variables are described in the terms of mean, range and standard deviation. Data was checked for normality before applying appropriate tests of significance.

Significance of difference in means was calculated using independent $t$ test. Significance of $\mathrm{p}$ value was taken as $\mathrm{p}<0.05$. Ethical permission was obtained from Institutional Ethics Committee of the hospital. The confidentiality of the study participants was maintained at all points of the study.

\section{RESULTS}

\section{Baseline characteristics}

Among the total $100(100 \%)$ study participants- there were two groups with each group consisted of 50. Group1 were those with AFI $>5$ and Group 2 with AFI <5. The 
age of the study participants varied between 20 years to 35 years. The mean (SD) age of study participants in group 1 and group 2 was $28( \pm 2.96)$ and $29( \pm 2.92)$ respectively. (Table 1 )

Mean (SD) hemoglobin of participants in Group 1 was $10.8( \pm 2.5)$ gms whereas it was $11.9( \pm 1.6)$ in group 2 . Random blood sugar values were 93 and $92 \mathrm{mg} / \mathrm{dl}$ in group1 and group 2 respectively.

Table 1: Baseline parameters of study participants. $(\mathbf{n}=100)$.

\begin{tabular}{|c|c|c|}
\hline Profile of study participants & $\begin{array}{l}\text { Group } 1 \\
\mathbf{N}=50 \\
\mathbf{N}(\%)\end{array}$ & $\begin{array}{l}\text { Group } 2 \\
\mathbf{N}=\mathbf{5 0} \\
\mathbf{N}(\%)\end{array}$ \\
\hline \multicolumn{3}{|l|}{ Age group } \\
\hline$\leq 20$ years & $1(2)$ & 0 \\
\hline $21-30$ years & $38(76)$ & $31(62)$ \\
\hline$>30$ years & $11(22)$ & $19(38)$ \\
\hline \multicolumn{3}{|l|}{ Gravida } \\
\hline Primigravida & $38(76)$ & $40(80)$ \\
\hline Multigravida & $12(24)$ & $10(20)$ \\
\hline \multicolumn{3}{|l|}{ Residence } \\
\hline Urban & $9(18)$ & $11(22)$ \\
\hline Rural & $41(82)$ & $39(78)$ \\
\hline
\end{tabular}

\section{Ultrasound features}

With ultrasound, certain parameters such as gestational age, amniotic fluid index, estimated fetal weight and placental position were determined.

Mean (SD) values of gestational age of group 1 and 2 were $31( \pm 2)$ and $32( \pm 2)$ respectively. Mean (SD) value of group 1 and 2 amniotic fluid indices was 10.2( \pm 2.2$)$ and $4.5( \pm 0.7)$ respectively. Estimated fetal weight (in kilogram) of group 1 and 2 was $2.6( \pm 0.23)$ and $2.0( \pm 0.29)$ respectively. Comparison of maternal outcomes such as latency period, chorioamnionitis, placental abruption and mode of delivery was done with two groups. Chorioamnionitis was present in $4(8 \%)$ of participants in group 1 and $6(12 \%)$ in group 2 . Latency period of $<48$ hours in $82 \%$ of study participants in group 1 and $92 \%$ of study participants. Latency period of $>48$ hours in $18 \%$ and $8 \%$ in group 1 and group 2 respectively.

Abruptio placenta was seen in $10 \%$ of study participants in group 1 and 14\% of study population in group 2 . Chorioamnionitis, abruption placenta and cord prolapsed were found to be higher in group $2(\mathrm{AFI}<5)$. Proportion of caesarean section was found to be much higher in group 2 when compared to group 1 . This was found to be significant $(\mathrm{P}$ value $=0.042)($ Table 2$)$.

Table 2: Distribution of study participants according to maternal outcomes. $(n=100)$.

\begin{tabular}{|c|c|c|c|c|}
\hline Maternal outcomes & Group 1, N=50, N (\%) & Group 2, N=50, N (\%) & OR $(95 \% \mathrm{CI})$ & P value \\
\hline \multicolumn{5}{|l|}{ Period of latency } \\
\hline$<48$ hours & $41(82)$ & $46(92)$ & \multirow{2}{*}{$0.396(0.11-1.38)$} & \\
\hline$>48$ hours & $9(18)$ & $4(8)$ & & 0.137 \\
\hline \multicolumn{3}{|l|}{ Chorioamnionitis } & \multirow{3}{*}{$1.00(0.32-3.08)$} & \multirow{3}{*}{0.121} \\
\hline Yes & $4(8)$ & $6(12)$ & & \\
\hline No & $46(92)$ & $44(88)$ & & \\
\hline \multicolumn{3}{|l|}{ Abruptio placenta } & \multirow{3}{*}{$0.683(0.20-2.31)$} & \multirow{3}{*}{0.583} \\
\hline Present & $5(10)$ & $7(14)$ & & \\
\hline Absent & $45(90)$ & $43(86)$ & & \\
\hline \multicolumn{3}{|c|}{ Meconium stained liquor } & \multirow{3}{*}{$0.243(0.01-1.78)$} & \multirow{3}{*}{0.346} \\
\hline Yes & $11(22)$ & $10(20)$ & & \\
\hline No & $39(78)$ & $40(80)$ & & \\
\hline \multicolumn{3}{|l|}{ Cord prolapse } & \multirow{3}{*}{$0.787(0.21-1.56)$} & \multirow{3}{*}{0.252} \\
\hline Yes & $1(2)$ & $2(4)$ & & \\
\hline No & $49(98)$ & $48(96)$ & & \\
\hline \multicolumn{3}{|l|}{ Mode of delivery } & \multirow{3}{*}{$2.068(1.70-6.11)$} & \multirow{3}{*}{0.042} \\
\hline Vaginal & $20(40)$ & $12(24)$ & & \\
\hline Caeserean section & $30(60)$ & $38(76)$ & & \\
\hline
\end{tabular}

Chi square test applied, $\mathrm{p}$ value $<0.05$ is significant

Comparison of neonatal outcomes such as NICU admission, RDS and neonatal sepsis was done with two groups. Among the neonatal outcomes, higher NICU admission was required in group 2 and this was found to be statistically significant. ( $p$ value $=0.001$ ). NICU admission was required in $18 \%$ of the neonates in Group 1 whereas $48 \%$ of neonates required NICU admission in Group 2. RDS developed in $12 \%$ and $8 \%$ of neonates in 
Group 1 and Group 2 respectively. Neonatal mortality among group 1 was $4 \%$ whereas in group 2 it was $2 \%$. Likewise, more than 60 days NICU stay was required in
$20 \%$ of group1 neonates. Upto 28 days admission in NICU was required in $24 \%$ of group 1 neonates and $48 \%$ of group 2 neonates (Table 3).

Table 3: Distribution of study participants according to neonatal outcomes. $(n=100)$.

\begin{tabular}{|c|c|c|c|c|}
\hline Neonatal outcomes & Group $1, \mathbf{N}=\mathbf{5 0}, \mathbf{N}(\%)$ & Group 2, N=50, N (\%) & OR $(95 \% \mathrm{CI})$ & p value \\
\hline \multicolumn{3}{|l|}{ NICU admission } & \multirow{3}{*}{$2.238(1.09-3.59)$} & \multirow{3}{*}{0.001} \\
\hline Yes & $9(18)$ & $24(48)$ & & \\
\hline No & $41(82)$ & $26(52)$ & & \\
\hline \multicolumn{3}{|c|}{ Duration of neonatal stay } & \multirow{5}{*}{-} & \multirow{5}{*}{0.560} \\
\hline 0-28 days & $12(24)$ & $24(48)$ & & \\
\hline $28-45$ days & $18(36)$ & $18(36)$ & & \\
\hline $46-60$ days & $10(20)$ & $8(16)$ & & \\
\hline$>60$ days & $10(20)$ & 0 & & \\
\hline \multicolumn{3}{|c|}{ Respiratory distress syndrom } & \multirow{3}{*}{$0.306(0.059-1.59)$} & \multirow{3}{*}{0.043} \\
\hline Yes & $4(8)$ & $6(12)$ & & \\
\hline No & $46(92)$ & $44(88)$ & & \\
\hline \multicolumn{3}{|l|}{ Neonatal sepsis } & \multirow{3}{*}{$1.397(0.44-4.367)$} & \multirow{3}{*}{0.128} \\
\hline Yes & $2(4)$ & $7(14)$ & & \\
\hline No & $48(96)$ & $43(86)$ & & \\
\hline \multicolumn{3}{|l|}{ Early neonatal death } & \multirow{3}{*}{$2.12(0.786-4.32)$} & \multirow{3}{*}{0.140} \\
\hline Yes & $2(4)$ & $1(2)$ & & \\
\hline No & $48(96)$ & $49(98)$ & & \\
\hline
\end{tabular}

Chi square test applied, $\mathrm{p}$ value $<0.05$ is significant

Mean $( \pm \mathrm{SD})$ of 1 -minute APGAR among group 1 was $7.84( \pm 0.58)$ and $5.5( \pm 1.41)$ in group 2 . Mean $( \pm S D)$ of 5minute APGAR among group 1 was $8.96( \pm 0.49)$ and $7.38( \pm 1.42)$ in group 2. APGAR scores at 1 and 5 minutes were compared between the two groups. It was found from the analysis that APGAR scores at 1 and 5 minutes were found to be lower among those with Group 2 when compared to those Group 1. This was found to be statistically significant. $(\mathrm{p}=0.000)(\mathrm{p}=0.000)($ Table 4$)$

Table 4: Comparison of mean APGAR scores among groups $(\mathrm{N}=100)$.

\begin{tabular}{|c|c|c|c|c|c|}
\hline Variables & Mean (SD) & t value & Mean Difference & $95 \% \mathrm{CI}$ & p value \\
\hline \multicolumn{6}{|c|}{ APGAR (1') } \\
\hline Group1 & $7.84( \pm 0.58)$ & \multirow{2}{*}{10.52} & \multirow{2}{*}{2.28} & \multirow{2}{*}{$1.850-2.710$} & \multirow{2}{*}{0.000} \\
\hline Group 2 & $5.5( \pm 1.41)$ & & & & \\
\hline \multicolumn{6}{|c|}{ APGAR (5') } \\
\hline Group 1 & $8.96( \pm 0.49)$ & \multirow{2}{*}{7.40} & \multirow{2}{*}{1.58} & \multirow{2}{*}{$1.156-2.004$} & \multirow{2}{*}{0.000} \\
\hline Group 2 & $7.38( \pm 1.42)$ & & & & \\
\hline
\end{tabular}

Independent $t$ test applied, $\mathrm{p}$ value $<0.05$ is significant

\section{DISCUSSION}

In the present study, $80 \%$ of study participants were from rural area and $20 \%$ were from urban area. PPROM was found frequently with low socioeconomic class and rural area. Higher chances of infections due to unhygienic living conditions in rural area is an independent risk factor for PPROM. ${ }^{13-14}$ PPROM was not age specific. Majority (69\%) belong to age group 21-30 years of age. Similar finding was reported by Gandhi $\mathrm{M}$ et al $(77.6 \%)$ and Kumari BR et al (49\%). $78 \%$ were primigravida in present study. This is similar to previous study finding as well- $60.7 \%, 58 \% .^{15,16}$

Chorioamnionitis was present in $6(12 \%)$ of participants in group2 and $4(8 \%)$ in group 1 . Overall incidence of chorioamninionitis in the study population was $20 \%$. In present study it was found that higher chorioamnionitis was found in group 2 when compared to group 1. Ekin A et al found that AFI $<5 \mathrm{~cm}$ demonstrated a significantly a 
higher rate of clinical chorioamnionitis. ${ }^{17} \mathrm{Yu}$ et al reported that $17.8 \%$ of pregnancies and Jiaswal AA et al reported clinical chorioamnionitis $(11.9 \%) .{ }^{18,19}$ Majority of the study participants required caesarean section. In Group $160 \%$ and in group 2, 76\% required caserean section for delivery of the baby. Overall, $70 \%$ had caesarean section in present study. In a study by Mohakar et al reported $65 \%$ patients had vaginal delivery and $25 \%$ required LSCS which is lower than present study findings. ${ }^{12}$

NICU admission was required in $18 \%$ of the neonates in Group 1 whereas $48 \%$ of neonates required NICU admission in Group 2. Totally in both groups the NICU admission was $66 \%$. Higher NICU admission in group 2 was found to be statistically significant. This is similar to previous study finding by $\mathrm{Yu}$ et al which reported $72.9 \%$ NICU admission. ${ }^{18}$ RDS developed in $12 \%$ and $8 \%$ of neonates in Group 1 and Group 2 respectively. Over all $10 \%$ had RDS in present study. In a study by Patil S et al twenty six percent newborn suffered from respiratory distress syndrome. ${ }^{20}$ RDS is not significantly associated with two groups. Likewise, in a study by Khanal $\mathrm{S}$ et al which reported that Respiratory system related problems like birth asphyxia, respiratory distress syndrome, apnea and pneumonia were common in both group but not statistically significant $(\mathrm{p}>0.05) .^{21}$

\section{CONCLUSION}

PPROM is associated with increased maternal and perinatal complications especially when $\mathrm{AFI}<5$. Caserean section rates, NICU admission, low mean APGAR scores and neonatal sepsis were found to be higher in PPROM with low amniotic fluid. Identification of risk factors, prompt treatment of infections and antibiotics following high vaginal swab culture might reduce the complications associated with PPROM. Prevention of risk factors and prediction of these morbidities are important in the management of PPROM.

Funding: No funding sources

Conflict of interest: None declared

Ethical approval: The study was approved by the Institutional Ethics Committee

\section{REFERENCES}

1. Beckmann, Charles. Obstetrics and Gynecology, 7e. Philadelphia: Wolters Kluwer Health/Lippincott Williams and Wilkins. pp. Chapter 17: Premature Rupture of Membranes. 2014;169-173.

2. Meis PJ, Ernest JM, Moore ML. Causes of low birth weight births in public and private patients. Am J Obstet Gynecol. 1987;156(5):1165-8.

3. Al-Riyami N, Al-Shezawi F, Al-Ruheili I, AlDughaishi T, Al-Khabori M. Perinatal Outcome in Pregnancies with Extreme Preterm Premature Rupture of Membranes (Mid-Trimester PROM). Sultan Qaboos Univ Med J. 2013;13(1):51-6.
4. Bendon RW, Faye-Petersen O, Pavlova Z, Qureshi F, Mercer B, Miodovnik M, et al. Fetal membrane histology in preterm premature rupture of membranes: comparison to controls, and between antibiotic and placebo treatment. Pediatr Dev Pathol. 1999;2(6):552-8.

5. Mercer B, Milluzzi C, Collin M. Periviable birth at 20 to 26 wk of gestation: proximate causes, previous obstetric history and recurrence risk. Am J Obstet Gynecol. 2005;193(3 Pt 2):1175-80.

6. Gonan R, Hannah ME, Milligan JE. Does prolonged premature rupture of the membranes predispose to abruption placenta? Obstet Gynecol. 1989;74(3):34750.

7. Ananth CC, Oyelese Y, Srinivas N, Yeo L, Vintzileos AM. Preterm premature rupture of membranes, intrauterine infection, and oligohydraminos: risk factors for placental abruption. Obstet Gynecol. 2004;104(1):71-7.

8. Mercer BM. Preterm premature rupture of the membranes. Obstet Gynecol. 2003;101(1):178-93.

9. Vintzileos AM, Campbell WA, Nochimson DJ. Degree oligohydramnios and pregnancy outcome in patients with PROM. Obstet Gynecol. 1985;66(2):162-7.

10. Mercer BM, Rabello YA, Thurnau GR. The NICHDMFMU antibiotic treatment of preterm PROM study: Impact of initial amniotic fliud volume on pregnancy outcome: NICHD- MFMU Network. Am J Obstet Gynecol. 2006;194(2):438-45.

11. Lewis DF, Robichaux AG, Jaekle RK, Expectant management of preterm premature rupture of membranes and non-vertex presentation: what are the risks? Am J Obstet Gynecol. 2007;196(6): 566e1-6.

12. Mohokar SA, Bava AK, Nandanwar YS. Analysis of Maternal and Perinatal Outcome in Cases of Preterm Premature Rupture of Membranes. Bombay Hospital J.2015;57(3):285-8.

13. Hargar JH, Hsing AW, Tuomala RE, Gibbs RS, Mead PB, Eschenbach DA, Knox GE, Polk BF, Risk Factors for preterm premature rupture of fetal membranes: a multicentric case control study. Am J Obstet Gynecol.1990:163(1):130-7.

14. Gomez R, Romero R, Edwin SS, David C Pathogenesis of preterm labor and preterm premature rupture of membranes associated with intrauterine infection. Infect Dis Clinic North Am.1997:11(1);135-76.

15. Gandhi M, Shah F, Panchal C. Obstetric Outcomes In Premature Rupture Of The Membrane (Prom). Inter J Gynecol Obstet. 2012;16(2):1-5.

16. Kumari BR, Sailaja C, Usha P. Foetomaternal outcome in cases of premature rupture of membrane (prom) at term: an experience in our institute. J. Evolution Med Dent Sci. 2016;5(64):4508-11.

17. Souza AS,Patriota AF,Guerra GV,de Melo BC,Santos AC,Torres junior AC. Amniotic fluid volume and maternal outcomes in women with preterm premature rupture of membranes. Rev Bras Gynecol Obstet. 2014;36(4):146-51. 
18. Yu H, Wang X, Gao H, You Y, Xing A. Perinatal outcomes of pregnancies complicated by preterm premature rupture of the membranes before 34 weeks of gestation in a tertiary center in China: a retrospective review. Biosci Trends. 2015;9(1):3541.

19. Jaiswal AA, Hariharan C, Dewani DKC. Study of maternal and fetal outcomes in premature rupture of membrane in central rural india. Int $\mathbf{J}$ Reprod Contracept Obstet Gynecol. 2017;6(4):1409-12.

20. Patil S, Patil V. Maternal and Foetal Outcome in Premature Rupture of Membranes. IOSR J Dent Med Sci .2014;13(12):56-83.
21. Khanal S, Zhang W, Rajbhandari Shrestha N, Dahal GR. A comparative study of outcome of preterm neonate with and without history of preterm premature rupture of membrane. Nepal Med Coll J. 2009;11(2):99-103.

Cite this article as: Britto JJ, Sailatha R, Nalini AP. Role of amniotic fluid index on maternal and neonatal outcomes among obstetric women with preterm premature rupture of membranes. Int $\mathbf{J}$ Reprod Contracept Obstet Gynecol 2018;7:4171-6. 